\title{
Segunda Sem Carne na Faculdade de Saúde Pública: um Projeto de Intervenção
}

\author{
Meatless Monday at Public Health School: \\ an Intervention Project
}

\section{RESUMO}

O consumo excessivo de carne, principalmente vermelha e processada, está relacionado com o aumento da incidência de doenças crônicas como câncer, diabetes, doenças cardiovasculares, excesso de peso e maior risco de mortalidade. Além do custo gerado à saúde, a produção da carne também gera grande impacto no meio ambiente. A partir dessas informações, elaborou-se o projeto Segunda Sem Carne na Faculdade de Saúde Pública. O trabalho tem por objetivo discutir os riscos causados à saúde e ao meio ambiente pelo consumo excessivo de carne bem como avaliar o impacto do mesmo na mudança do hábito alimentar da população estudada. A iniciativa contou com a divulgação de dados sobre consumo excessivo de carne, seus impactos na saúde e no meio ambiente, propondo a inclusão de um cardápio sem carne em uma segunda feira de cada mês do período letivo do ano de 2012 no restaurante da Faculdade de Saúde Pública da Universidade de São Paulo/COSEAS. Para avaliar a eficácia da iniciativa, foram realizadas três pesquisas de opinião com os comensais do restaurante ao longo do projeto, sendo uma online e duas presenciais. Sete meses após o início da intervenção, $73 \%$ dos pesquisados disseram estar mais conscientes sobre os malefícios associados ao consumo excessivo de carne; $42 \%$ disseram que diminuíram o consumo de carne devido aos esclarecimentos fornecidos pelo projeto.

Palavras-chave: Consumo de Carne. Saúde. Projeto de Intervenção

\section{ABSTRACT}

Excessive meat consumption, especially red and processed meat, is related to the increase in the incidence of chronic diseases such as cancer, diabetes, cardiovascular diseases, overweight and increased risk of mortality. Besides the cost generated to health, beef production also generates impacts on the environment. Thus, the Meatless Monday Project was designed at the School of Public Health, in order to discuss the risks

BRUNA LACERDA,

Aline Carvalho,

Joyce Martins,

Camila Negrão,

Soraya Selem, Regina

Fisberg E DirCe

MARCHIONI

Universidade de São Paulo. Faculdade de Saúde Pública, São Paulo, Brasil 
caused by excessive meat consumption. Data about excessive meat consumption, health and environment impacts were offered to population and a no-meat menu included on one Monday of each month within the period of the academic year of 2012 in the restaurant of the School of Public Health, University of São Paulo/Superintendence of Social Welfare. Three polls were performed to evaluate the effectiveness of the initiative throughout the project, the first was done online and the others were interviewer-interviewee. Seven months after the intervention, $73 \%$ of people said they were more aware about the harms associated with excessive consumption of meat; $42 \%$ reduced meat consumption due to the clarifications provided by the project.

Keywords: Meat Consumption. Health. Intervention Project.

\section{INTRODUÇÃO}

A prevalência de doenças crônicas tem aumentado progressivamente nas últimas décadas, tornando-se um grave problema de saúde pública [9]. Há evidências da associação entre o consumo excessivo de carne, principalmente vermelha e processada, e doenças cardiovasculares, além de diabetes [11], câncer de cólon e reto [16], ganho de peso $[7,15]$ e maior risco de mortalidade $[7,15]$. Estudos mostram que o consumo de $50 g$ de carne processada pode aumentar o risco em $42 \%$ de doenças cardiovasculares e $19 \%$ de diabetes [11].

A carne sempre teve grande importância na alimentação humana devido à sua composição, com quantidade significativa de proteína, minerais, vitaminas e ácidos graxos [10]. Atualmente, as diretrizes brasileiras orientam a ingestão de uma porção de carne por dia, equivalente a $190 \mathrm{kcal}$, como parte de uma dieta saudável para a população [12]. Porém, o que se tem percebido é um aumento progressivo desse consumo [4].

Dados da Pesquisa de Orçamentos Familiares (POF) evidenciam que a carne está inserida no grupo de alimentos que lidera os gastos com alimentação no país (21,9\%), nas áreas urbana (21,3\%) e rural (25,2\%). Comparando os resultados de 2002/03 e 2008/o9, é possível observar um aumento no percentual de gastos de $18,3 \%$ para $21,9 \%$ com esse tipo de alimento [8].

Em um estudo de base populacional realizado em São Paulo, observou-se que o consumo de carnes aumentou cerca de $20 \%$ de 2003 para 2008 [5]. O consumo médio de carne total em São Paulo (163g) foi superior aos encontrados em diversos países como Irlanda (138g), Espanha (135g), Holanda (125g) e Alemanha (120g) [10]. Para carne processada, o consumo do paulistano (33g) [5] foi superior ao da Irlanda (25g), Grécia (8g) e Itália (27g) [10].

Além do custo à saúde gerado pelo consumo excessivo de carne, a sua produção também gera impacto sobre o meio ambiente, pois promove o desmatamento [12], ocupando e degradando vastas áreas de terra, resultando no aumento do efeito estufa, na redução da biodiversidade, na reposição de água doce, no estímulo à compactação do solo, o que reduz a infiltração da água, resultando no aumento do escoamento e em perda de nutrientes do solo $[1,14]$.

A pecuária é o setor responsável por emitir cerca de $18 \%$ dos gases de efeito estufa 
do mundo [12]. Para a produção de um quilograma $(\mathrm{kg})$ de carne bovina são gerados cerca de $44 \mathrm{~kg}$ de gases de efeito estufa [6]. O impacto ambiental gerado pela produção de carne para abastecer o município de São Paulo foi estimado em 18 milhões de toneladas de equivalentes de $\mathrm{CO}_{2}$ em 2003 [6].

Pensando nos efeitos que a produção e o consumo de carne causam na saúde e no meio ambiente, elaborou-se o projeto de extensão "Segunda Sem Carne na FSP” (SSC na FSP) em parceria com a Superintendência de Assistência Social da USP (SAS). O principal objetivo do projeto é discutir a relação entre o consumo excessivo de carne e o risco à saúde e ao meio ambiente.

\section{OBJETIVO}

Avaliar o impacto do projeto de extensão universitária Segunda Sem Carne na FSP.

\section{METODOLOGIA}

O projeto de extensão Segunda Sem Carne na FSP consiste em uma intervenção na comunidade da Faculdade de Saúde Pública da USP a partir da divulgação de dados sobre o consumo excessivo de carne e seus impactos na saúde e no meio ambiente, por meio de vídeos; site do projeto; palestras; boletins informativos; mídias sociais; entrevistas a jornais, revistas, rádios, canais de televisão; e, durante o ano letivo de 2012, introdução de um cardápio sem carne em uma segunda-feira de cada mês no Restaurante Universitário da Faculdade de Saúde Pública.

Foram realizadas três pesquisas de opinião com os comensais ao longo do projeto, sendo uma online e duas presenciais.

A primeira pesquisa foi realizada em março de 2012 e teve como objetivo avaliar a aceitabilidade do novo cardápio, estimar a proporção de pessoas que se preocupavam com o tema e estavam dispostas a realizar mudanças em seus hábitos alimentares, além de saber como os materiais educativos e o site melhoraram o entendimento sobre o tema. Para tal, foi aplicado um questionário online.

A segunda pesquisa foi realizada em outubro de 2012 e teve como objetivo avaliar a aceitação do projeto. Foi utilizada uma urna montada na saída do restaurante, em que era feita a pergunta: “O que você acha do projeto Segunda Sem Carne na FSP?", utilizando-se os cartões nas cores vermelha, amarela e verde como resposta, que representaram "não gosto", "indiferente” e "gosto", respectivamente. Também foram disponibilizados cartões nos quais os participantes da pesquisa puderam deixar seus comentários, críticas, dúvidas, elogios e sugestões.

A terceira pesquisa foi realizada em novembro de 2012 e teve como objetivo avaliar se o projeto Segunda Sem Carne na FSP estava conseguindo conscientizar os usuários do restaurante universitário SAS/USP sobre os malefícios do consumo excessivo de carne e se eles haviam diminuído seu consumo de carne a partir das informações fornecidas. Para tal, foi aplicado um questionário na saída do restaurante, com as perguntas: 
"A partir do projeto você percebeu que o consumo excessivo de carne pode trazer malefícios para saúde e meio ambiente? Você diminuiu seu consumo de carne a partir das informações oferecidas pelo projeto?”

\section{RESULTADOS E DISCUSSÃO}

Na primeira pesquisa, registraram-se 156 respostas, sendo que a maior parte dos participantes eram alunos da graduação (48\%), seguidos dos alunos da pós-graduação (29\%), funcionários ( $13 \%)$, visitantes $(7 \%)$ e docentes (3\%). Verificou-se que $92 \%$ das respostas foram positivas quando os comensais foram questionados sobre a iniciativa do projeto e $73 \%$ alegaram que, a partir das informações fornecidas pela campanha, pretendiam diminuir seu consumo de carne.

Verificou-se que $58 \%$ das pessoas que responderam a primeira pesquisa acessaram o site e $87,5 \%$ acharam que o conteúdo do site contribuiu para esclarecimento dos objetivos do projeto, sendo que, dessas, $34 \%$ acharam que o site contribuiu muito.

A segunda pesquisa aconteceu presencialmente no dia 22 de outubro de 2012. Cerca de 190 pessoas responderam a pesquisa e $78 \%$ dos participantes alegaram gostar do projeto.

$\mathrm{Na}$ terceira pesquisa realizada foram obtidas 120 respostas. Verificou-se que $73 \% \mathrm{dos}$ pesquisados disseram estar mais conscientes sobre os malefícios associados ao consumo excessivo de carne devido as informações oferecidas pelo projeto, e $42 \%$ afirmaram ter diminuído o consumo de carne após o início da intervenção.

Em relação à divulgação do projeto, a página do Facebook alcançou usuários de mais de 20 países, como Estados Unidos, França, Reino Unido, Portugal, Argentina, entre outros. No Brasil foram atingidas diversas cidades das regiões Nordeste, Sul, Sudeste e Centro-Oeste do país e a página recebeu cerca de 600 visitas diretas e 4.000 indiretas nesse período. O site alcançou mais de 2.000 acessos.

Observou-se que essas formas de divulgação constituíram um caminho mais imediato e abrangente para intensificar a divulgação científica para a comunidade FSP, além de atrair a atenção de mais pessoas para o assunto em questão, tornando comuns informações de caráter inter e transdisciplinar. $\mathrm{O}$ projeto também foi divulgado por meio de entrevistas para jornais, rádios, revistas e sites como Rádio Estadão, CBN, Jovem Pan, além TV USP, TV Cultura, Revista Vida e Saúde, Jornal do Campus entre outras. Além de ser produzido um vídeo na forma de desenho animado stop-motion para divulgar o projeto aos comensais.

Existem projetos similares ao Segunda Sem Carne na FSP, como na Alimentação Escolar do município de São Paulo. Desde 2011, a Secretaria do Verde e Meio Ambiente e a Secretaria de Educação do município propuseram um projeto de merenda escolar sem carne duas vezes ao mês a crianças e adolescentes da rede pública. Atualmente, cerca de 625 mil alunos recebem refeições sem carne quinzenalmente [13]. Posteriormente, outras cidades também aderiram à campanha, como Curitiba [2], Piracicaba, Recife, Niterói e Osasco [13,3].

Apesar da alteração do cardápio da alimentação escolar da rede pública de alguns municípios brasileiros, poucas atividades educativas foram realizadas com o intuito de explicar o projeto e mostrar o possível impacto da mudança na alimentação, na saúde e 
no meio ambiente. Observou-se, no presente trabalho, que o uso de materiais educativos e formas diferentes de divulgação ampliaram a discussão sobre o tema, e puderam promover mudança do hábito alimentar em parte da população estudada.

\section{CONCLUSÃO}

Verificou-se que o projeto Segunda Sem Carne na FSP foi bem avaliado pela comunidade que participou das pesquisas. Além disso, a divulgação sobre os efeitos do consumo excessivo de carne foi efetiva, visto que quase metade dos entrevistados na terceira pesquisa alegou ter diminuído o consumo de carne a partir das informações fornecidas pelo projeto.

Esse fato é importante, pois o elevado consumo de carne tem um impacto direto e indireto sobre a saúde e o meio ambiente. O Segunda Sem Carne na FSP é um importante passo para aproximar a pesquisa, produzida pela Universidade, da população e incentivar ações que podem melhorar a qualidade de vida dos indivíduos a longo prazo.

\section{REFERÊNCIAS}

[1] BLEIL, S. I. O padrão alimentar ocidental: considerações sobre a mudança de hábitos no Brasil. Cadernos de Debate, Campinas, v. 6, p. 1-25, 1998.

[2] CÂMARA MUNICIPAL DE CURITIBA. Notícias do legislativo. Disponível em: $<$ http://www.cmc.pr.gov.br/ass_det.php?not=18788>. Acesso em: 5 maio 2013.

[3] CÂMARA MUNICIPAL DE SANTOS. Notícias. Disponível em: <http://www. camarasantos.sp.gov.br/noticia.asp? codigo $=4069 \& C O D \_M E N U=185>$. Acesso em: 5 maio 2013 .

[4] CARVALHO A. M. et al. Excessive meat consumption in Brazil: diet quality and environmental impacts. Pub Health Nutr., 16, p. 1893-1899, 2012. Disponível em: <http://journals.cambridge.org/action/displayAbstract?fromPage=online \&aid=8991139>. Acesso em: 10 ago. 2013. <DOI: 10.1017/S1368980012003916>

[5] CARVALHO, A. M. Tendência temporal do consumo de carne no Município de São Paulo: estudo de base populacional - ISA Capital 2003/2008. São Paulo, 2012. Dissertação (Mestre em Nutrição em Saúde Pública) - Faculdade de Saúde Pública, Universidade de São Paulo, São Paulo, 2012.

[6] CEDEBERG, C. et al. Including carbon emissions from deforestation in the carbon footprint of Brazilian beef. Environ Sci Technol., v. 45, p. 1773-1779, 2011. $<$ dx.doi.org/10.1021/es103240z>

[7] HODGSON, J. M. et al. Increased lean red meat intake does not elevate markers of oxidative stress and inflammation in humans. J Nutr., v. 137, p. 363-367, 2007.

[8] INSTITUTO BRASILEIRO DE GEOGRAFIA E ESTATÍSTICA (IBGE). Indicadores IBGE: Pesquisa de orçamentos familiares 2008-2009: despesas, rendimentos e condições de vida. Rio de Janeiro: IBGE; 2010.

[9] LIMA, F. E. L. et al. Ácidos graxos e doenças cardiovasculares: uma revisão. Rev. Nutr., v. 13, p. 73-80, 2010. 
[10] MCAFEE, A.J. et al. Red meat consumption: An overview of the risks and benefits. Meat Scienc., v. 84, p. 1-13, 2010. < http://dx.doi.org/10.1016/j. meatsci.2009.08.029>

[11] MICHA, R.; Wallace, S. K.; Mozaffarian, D. Red and Processed Meat Consumption and Risk of Incident Coronary Heart Disease, Stroke, and Diabetes Mellitus: A systematic Review and Meta-Analysis. Circulation., v. 121, p. 2271-2283, 2010. < http://dx.doi.org/10.1161/CIRCULATIONAHA.109.924977>

[12] MINISTÉRIO DA SAÚDE. Secretaria de Atenção à Saúde. Coordenação-Geral da Política de Alimentação e Nutrição. Guia alimentar para a população brasileira: promovendo a alimentação saudável. Brasília: Ministério da Saúde, 2006.

[13] SEGUNDA SEM CARNE. Notícias. Disponível em: <http://www.segundasemcarne.com.br/2011/12/26/escolas-municipais-de-sao-paulo-introduzem-refeicoes-sem-carne-na-merenda/>. Acesso em: 5 maio 2013.

[14] STEINFELD, H. et al. Livestock's long shadow: environmental issues and options. Food and Agriculture Organization of the United Nations: Roma, 2006.

[15] VERGNAUD, A. C. et al. Meat consumption and prospective weight change in participants of the EPIC-PANACEA study. Am J Clin Nutr., v. 92, p. 398-407, 2010. < http://dx.doi.org/10.3945/ajcn.2009.28713>

[16] WORLD CANCER RESEARCH FUND. / American Institute for Cancer Research. Food, Nutrition, Physical Activity, and the Prevention of Cancer: a Global Perspective. Washington (DC): AICR, 2007.

\section{AGRADECIMENTOS}

Agradecemos a toda equipe da Superintendência de Assistência Social da USP (SAS-USP), nutricionistas e funcionários do Restaurante Universitário da Faculdade de Saúde Pública, alunos de graduação e funcionários do TIC/FSP-USP, aprimorandas e nutricionistas do CRNutri pelo apoio e ajuda para realizar o projeto.

À diretora da FSP-USP, Helena Ribeiro, e ao departamento de Nutrição da FSP-USP pelo apoio institucional.

Ao Segunda Sem Carne Brasil pelo apoio e discussões.

A LIN E CARVA LHO doutoranda do Programa de Nutrição em Saúde Pública do Departamento de Nutrição da Faculdade de Saúde Pública da Universidade de São Paulo (FSP-USP) - e-mail: alinenutri@usp.br

BRUNA LACERDA graduanda em Nutrição da Faculdade de Saúde Pública da Universidade de São Paulo (FSP-USP)

JOYCE MARTINS graduanda em Nutrição da Faculdade de Saúde Pública da Universidade de São Paulo (FSP-USP) 
CAMILA NEGRÃo bacharel em Nutrição da Faculdade de Saúde Pública da Universidade de São Paulo (FSP-USP)

SORAYA SE Lem mestre em Ciências do Programa de Nutrição em Saúde Pública do Departamento de Nutrição da Faculdade de Saúde Pública da Universidade de São Paulo (FSP-USP)

REGINA FISBERG pesquisadora e professora associada do Departamento de Nutrição da Faculdade de Saúde Pública da Universidade de São Paulo (FSP-USP)

DIRCE MARCHIONI pesquisadora e professora associada do Departamento de Nutrição da Faculdade de Saúde Pública da Universidade de São Paulo (FSP-USP) 